Asia Pacific Journal of Mathematics, Vol. 5, No. 1 (2018), 1-13

ISSN 2357-2205

\title{
THE INTUITIONISTIC TRIPLE $\chi$ OF IDEAL FUZZY REAL NUMBERS OVER $p$-METRIC SPACES DEFINED BY MUSIELAK ORLICZ FUNCTION
}

\author{
VANDANA $^{1}$, DEEPMALA $^{2}$, N. SUBRAMANIAN ${ }^{3}$ AND VISHNU NARAYAN MISHRA ${ }^{4,5, *}$
}

${ }^{1}$ Department of Management Studies, Indian Institute of Technology, Madras, Chennai 600 036, Tamil Nadu, India

${ }^{2}$ Mathematics Discipline, PDPM Indian Institute of Information Technology, Design and Manufacturing, Jabalpur, Dumna Airport Road, P.O.: Khamaria, Jabalpur 482 005, Madhya Pradesh, India

${ }^{3}$ Department of Mathematics, SASTRA University, Thanjavur-613 401, India

${ }^{4}$ Department of Mathematics,Indira Gandhi National Tribal University, Lalpur, Amarkantak, Anuppur 484 887, Madhya Pradesh, India

${ }^{5}$ L. 1627 Awadh Puri Colony Beniganj, (Phase-III), Opp.-I.T.I. Ayodhya Main Road, Faizabad-224 001, Uttar Pradesh, India

*Corresponding author: vishnunarayanmishra@gmail.com

Received June 13, 2016

\begin{abstract}
In this article we introduce the intuitionistic sequence spaces $\left[\chi_{f(\mu, \eta)}^{3 F I},\left\|\left(d\left(x_{1}\right), d\left(x_{2}\right), \cdots, d\left(x_{n-1}\right)\right)\right\|_{p}\right]$ and $\left[\Lambda_{f(\mu, \eta)}^{3 F I},\left\|\left(d\left(x_{1}\right), d\left(x_{2}\right), \cdots, d\left(x_{n-1}\right)\right)\right\|_{p}\right]$, and study some basic topological and algebraic properties of these spaces. Also we investigate the relations related to these spaces and some of their properties like solidity, symmetricity, convergence free etc., and also investigate some inclusion relations related to these spaces.

2010 Mathematics Subject Classification. 40A05; 40C05; 46A45; $03 \mathrm{E} 72$.

Key words and phrases. analytic sequence; double sequences; $\chi^{3 F I}$ space, Musielak - Orlicz function; intuitionistic fuzzy $p$ - metric space,fuzzy number; solid space, symmetricity; convergence free; sequence algebra,ideal.
\end{abstract}

\section{INTRODUCTION}

Throughout $w, \Gamma$ and $\Lambda$ denote the classes of all, entire and analytic scalar valued single sequences, respectively.

We can represent triple sequences by matrix. In case of double sequences we write in the form of a square. In the case of a triple sequence it will be in the form of a box in three dimensional case.

Some initial work on double series is found in Apostol [1] and double sequence spaces is (c)2018 Asia Pacific Journal of Mathematics 
found in Hardy [7], Subramanian et al. [8-14], and many others. Later on investigated by some initial work on triple sequence spaces is found in Sahiner et al. [15], Esi et al. [2-6], Subramanian et al. [16-25] and others [31-33].

Let $\left(x_{m n k}\right)$ be a triple sequence of real or complex numbers. Then the series $\sum_{m, n, k=1}^{\infty} x_{m n k}$ is called a triple series. The triple series $\sum_{m, n, k=1}^{\infty} x_{m n k}$ give one space is said to be convergent if and only if the triple sequence $\left(S_{m n k}\right)$ is convergent, where

$$
S_{m n k}=\sum_{i, j, q=1}^{m, n, k} x_{i j q}(m, n, k=1,2,3, \ldots) .
$$

A sequence $x=\left(x_{m n k}\right)$ is said to be triple analytic if

$$
\sup _{m, n, k}\left|x_{m n k}\right|^{\frac{1}{m+n+k}}<\infty .
$$

The vector space of all triple analytic sequences are usually denoted by $\Lambda^{3}$. A sequence $x=\left(x_{m n k}\right)$ is called triple entire sequence if

$$
\left|x_{m n k}\right|^{\frac{1}{m+n+k}} \rightarrow 0 \text { as } m, n, k \rightarrow \infty .
$$

The vector space of all triple entire sequences are usually denoted by $\Gamma^{3}$. Let the set of sequences with this property be denoted by $\Lambda^{3}$ and $\Gamma^{3}$ is a metric space with the metric

$$
d(x, y)=\sup _{m, n, k}\left\{\left|x_{m n k}-y_{m n k}\right|^{\frac{1}{m+n+k}}: m, n, k: 1,2,3, \ldots\right\},
$$

forall $x=\left\{x_{m n k}\right\}$ and $y=\left\{y_{m n k}\right\}$ in $\Gamma^{3}$. Let $\phi=\{$ finite sequences $\}$.

Consider a triple sequence $x=\left(x_{m n k}\right)$. The $(m, n, k)^{t h}$ section $x^{[m, n, k]}$ of the sequence is defined by $x^{[m, n, k]}=\sum_{i, j, q=0}^{m, n, k} x_{i j q} \delta_{i j q}$ for all $m, n, k \in \mathbb{N}$, where $\delta_{m n k}$ is a three dimensional matrix with 1 in the $(m, n, k)^{t h}$ position and zero otherwise.

Let $M$ and $\Phi$ are mutually complementary Orlicz functions. Then, we have:

(i) For all $u, y \geq 0$,

$$
u y \leq M(u)+\Phi(y),\left(Y_{\text {oung }}^{\prime} \text { s inequality }\right)[\text { See }[26]]
$$

(ii) For all $u \geq 0$,

$$
u \eta(u)=M(u)+\Phi(\eta(u)) .
$$

(iii) For all $u \geq 0$, and $0<\lambda<1$,

$$
M(\lambda u) \leq \lambda M(u)
$$

Lindenstrauss and Tzafriri [27] used the idea of Orlicz function to construct Orlicz sequence space

$$
\ell_{M}=\left\{x \in w: \sum_{k=1}^{\infty} M\left(\frac{\left|x_{k}\right|}{\rho}\right)<\infty, \text { for some } \rho>0\right\}
$$


The space $\ell_{M}$ with the norm

$$
\|x\|=\inf \left\{\rho>0: \sum_{k=1}^{\infty} M\left(\frac{\left|x_{k}\right|}{\rho}\right) \leq 1\right\},
$$

becomes a Banach space which is called an Orlicz sequence space. For $M(t)=t^{p}(1 \leq p<\infty)$, the spaces $\ell_{M}$ coincide with the classical sequence space $\ell_{p}$.

A sequence $f=\left(f_{m n k}\right)$ of Orlicz function is called a Musielak-Orllicz function. A sequence $g=\left(g_{m n k}\right)$ defined by

$$
g_{m n k}(v)=\sup \left\{|v| u-\left(f_{m n k}\right)(u): u \geq 0\right\}, m, n, k=1,2, \cdots
$$

is called the complementary function of a Musielak-Orlicz function $f$. For a given Musielak Orlicz function $f$, the Musielak-Orlicz sequence space $t_{f}$ is defined as follows

$$
t_{f}=\left\{x \in w^{3}: M_{f}\left(\left|x_{m n k}\right|\right)^{1 / m+n+k} \rightarrow 0 \text { as } m, n, k \rightarrow \infty\right\},
$$

where $M_{f}$ is a convex modular defined by

$$
M_{f}(x)=\sum_{m=1}^{\infty} \sum_{n=1}^{\infty} \sum_{k=1}^{\infty} f_{m n k}\left(\left|x_{m n k}\right|\right)^{1 / m+n+k}, x=\left(x_{m n k}\right) \in t_{f} .
$$

We consider $t_{f}$ equipped with the Luxemburg metric

$$
d(x, y)=\sup _{m n k}\left\{\inf \left(\sum_{m=1}^{\infty} \sum_{n=1}^{\infty} \sum_{k=1}^{\infty} f_{m n k}\left(\frac{\left|x_{m n k}\right|^{1 / m+n+k}}{m n k}\right)\right) \leq 1\right\}
$$

If $X$ is a sequence space, we give the following definitions:

(i) $X^{\prime}=$ the continuous dual of $X$;

(ii) $X^{\alpha}=\left\{a=\left(a_{m n k}\right): \sum_{m, n, k=1}^{\infty}\left|a_{m n k} x_{m n k}\right|<\infty\right.$, for each $\left.x \in X\right\}$;

(iii) $X^{\beta}=\left\{a=\left(a_{m n k}\right): \sum_{m, n, k=1}^{\infty} a_{m n k} x_{m n k}\right.$ is convegent, for each $\left.x \in X\right\}$;

(iv) $X^{\gamma}=\left\{a=\left(a_{m n k}\right): \sup _{m n k \geq 1}\left|\sum_{m, n, k=1}^{M, N, K} a_{m n k} x_{m n k}\right|<\infty\right.$, for each $\left.x \in X\right\}$;

(v)let $X$ be an $F K-$ space $\supset \phi$; then $X^{f}=\left\{f\left(\Im_{m n k}\right): f \in X^{\prime}\right\}$;

$(\mathrm{vi}) X^{\delta}=\left\{a=\left(a_{m n k}\right): \sup _{m n k}\left|a_{m n k} x_{m n k}\right|^{1 / m+n+k}<\infty\right.$, for each $\left.x \in X\right\}$;

$X^{\alpha}, X^{\beta}, X^{\gamma}$ are called $\alpha-($ orKöthe - Toeplitz)dual of $X, \beta$ - (or generalized - Köthe Toeplitz) dual of $X, \gamma-$ dual of $X, \delta-$ dual of $X$ respectively.

The notion of difference sequence spaces (for single sequences) was introduced by Kizmaz [28] as follows

$$
Z(\Delta)=\left\{x=\left(x_{k}\right) \in w:\left(\Delta x_{k}\right) \in Z\right\}
$$


for $Z=c, c_{0}$ and $\ell_{\infty}$, where $\Delta x_{k}=x_{k}-x_{k+1}$ for all $k \in \mathbb{N}$.

Later on the notion was further investigated by many others. We now introduce the following difference double sequence spaces defined by

$$
Z(\Delta)=\left\{x=\left(x_{m n}\right) \in w^{2}:\left(\Delta x_{m n}\right) \in Z\right\}
$$

where $Z=\Lambda^{2}, \chi^{2}$ and $\Delta x_{m n}=\left(x_{m n}-x_{m n+1}\right)-\left(x_{m+1 n}-x_{m+1 n+1}\right)=x_{m n}-x_{m n+1}-x_{m+1 n}+$ $x_{m+1 n+1}$ for all $m, n \in \mathbb{N}$.

Let $w^{3}, \chi^{3}\left(\Delta_{m n k}\right), \Lambda^{3}\left(\Delta_{m n k}\right)$ be denote the spaces of all, triple gai difference sequence space and triple analytic difference sequence space respectively and is defined as

$\Delta^{m} x_{m n}=\Delta \Delta^{m-1} x_{m n}=$

$\Delta^{m-1} x_{m n}-\Delta^{m-1} x_{m n+1}-\Delta^{m-1} x_{m n+2}-\Delta^{m-1} x_{m+1 n}-\Delta^{m-1} x_{m+1 n+1}-\Delta^{m-1} x_{m+1 n+2}-$ $\Delta^{m-1} x_{m+2 n}-\Delta^{m-1} x_{m+2 n+1}-\Delta^{m-1} x_{m+2 n+2}$

\section{Definition And Preliminaries}

A triple sequence $x=\left(x_{m n k}\right)$ has limit 0 (denoted by $\left.P-\lim x=0\right)$

(i.e) $\left((m+n+k) !\left|x_{m n k}\right|\right)^{1 / m+n+k} \rightarrow 0$ as $m, n, k \rightarrow \infty$. We shall write more briefly as $P-$ convergent to 0 .

2.1. Definition. A binary operation $*:[0,1] \times[0,1] \times[0,1] \rightarrow[0,1] \times[0,1] \times[0,1]$ is said to be continuous with metric if it satisfies the following conditions:

(1) $*$ is associative and commutative, (b) * continuous, (c) $a * 1=a$ for all $a \in[0,1]$, (d) $a * c \leq b * d$ whenever $a \leq b$ and $c \leq d$ for each $a, b, c, d \in[0.1]$. For example $d(a, b)=$ $a * b=a \cdot b=d(b, a)$.

2.2. Definition. A binary operation $\delta:[0,1] \times[0,1] \times[0,1] \rightarrow[0,1] \times[0,1] \times[0,1]$ is said to be continuous with co-metric if it satisfies the following conditions:

(1) $\delta$ is associative and commutative, (b) $\delta$ continuous, (c) $a \delta 0=a$ for all $a \in[0,1]$, (d) $a \delta c \leq b \delta d$ whenever $a \leq b$ and $c \leq d$ for each $a, b, c, d \in[0.1]$.

2.3. Note. The five tuple $(X, \mu, \eta, *, \delta)$ is said to be an intuitionistic fuzzy metric space(for short, IFMS) if $X$ is a vector space, $*$ is a continuous metric , $\delta$ is a continuous co-metric and $\mu, \eta$ are fuzzy sets on $X \times X \times X \times(0, \infty) \times(0, \infty) \times(0, \infty)$.

2.4. Definition. A Orlicz function was introduced by Nakano [29]. We recall that a Orlicz $f$ is a function from $[0, \infty) \rightarrow[0, \infty)$, such that

(1) $f(x)=0$ if and only if $x=0$

(2) $f(x+y) \leq f(x)+f(y)$, for all $x \geq 0, y \geq 0$,

(3) $f$ is increasing, 
(4) $f$ is continuous from the right at 0 . Since $|f(x)-f(y)| \leq f(|x-y|)$, it follows from here that $f$ is continuous on $[0, \infty)$. Let $n \in \mathbb{N}$ and $X$ be a real vector space of dimension $m$, where $n \leq m$. A real valued function $d_{p}\left(x_{1}, \ldots, x_{n}\right)=\left\|\left(d_{1}\left(x_{1}\right), \ldots, d_{n}\left(x_{n}\right)\right)\right\|_{p}$ on $X$ satisfying the following four conditions:

(i) $\left\|\left(d_{1}\left(x_{1}\right), \ldots, d_{n}\left(x_{n}\right)\right)\right\|_{p}=0$ if and and only if $d_{1}\left(x_{1}\right), \ldots, d_{n}\left(x_{n}\right)$ are linearly dependent,

(ii) $\left\|\left(d_{1}\left(x_{1}\right), \ldots, d_{n}\left(x_{n}\right)\right)\right\|_{p}$ is invariant under permutation,

(iii) $\left\|\left(\alpha d_{1}\left(x_{1}\right), \ldots, \alpha d_{n}\left(x_{n}\right)\right)\right\|_{p}=|\alpha|\left\|\left(d_{1}\left(x_{1}\right), \ldots, d_{n}\left(x_{n}\right)\right)\right\|_{p}, \alpha \in \mathbb{R}$

(iv) $d_{p}\left(\left(x_{1}, y_{1}\right),\left(x_{2}, y_{2}\right) \cdots\left(x_{n}, y_{n}\right)\right)=\left(d_{X}\left(x_{1}, x_{2}, \cdots x_{n}\right)^{p}+d_{Y}\left(y_{1}, y_{2}, \cdots y_{n}\right)^{p}\right)^{1 / p}$ for $1 \leq p<$ $\infty ;($ or $)$

(v) $d\left(\left(x_{1}, y_{1}\right),\left(x_{2}, y_{2}\right), \cdots\left(x_{n}, y_{n}\right)\right):=\sup \left\{d_{X}\left(x_{1}, x_{2}, \cdots x_{n}\right), d_{Y}\left(y_{1}, y_{2}, \cdots y_{n}\right)\right\}$,

for $x_{1}, x_{2}, \cdots x_{n} \in X, y_{1}, y_{2}, \cdots y_{n} \in Y$ is called the $p$ product metric of the Cartesian product of $n$ metric spaces is the $p$ norm of the $n$-vector of the norms of the $n$ subspaces.

A trivial example of $p$ product metric of $n$ metric space is the $p$ norm space is $X=\mathbb{R}$ equipped with the following Euclidean metric in the product space is the $p$ norm:

$$
\begin{array}{r}
\left\|\left(d_{1}\left(x_{1}\right), \ldots, d_{n}\left(x_{n}\right)\right)\right\|_{E}=\sup \left(\left|\operatorname{det}\left(d_{m n}\left(x_{m n}\right)\right)\right|\right)= \\
\sup \left(\left|\begin{array}{cccc}
d_{11}\left(x_{11}\right) & d_{12}\left(x_{12}\right) & \ldots & d_{1 n}\left(x_{1 n}\right) \\
d_{21}\left(x_{21}\right) & d_{22}\left(x_{22}\right) & \ldots & d_{2 n}\left(x_{1 n}\right) \\
\cdot & & & \\
\cdot & & & \\
\cdot & & & \\
d_{n 1}\left(x_{n 1}\right) & d_{n 2}\left(x_{n 2}\right) & \ldots & d_{n n}\left(x_{n n}\right)
\end{array}\right|\right)
\end{array}
$$

where $x_{i}=\left(x_{i 1}, \cdots x_{i n}\right) \in \mathbb{R}^{n}$ for each $i=1,2, \cdots n$.

If every Cauchy sequence in $X$ converges to some $L \in X$, then $X$ is said to be complete with respect to the $p$ - metric. Any complete $p$ - metric space is said to be $p-$ Banach metric space.

2.5. Definition. A family $I \subset 2^{Y \times Y \times Y}$ of subsets of a non empty set $Y$ is said to be an ideal in $Y$ if

(1) $\phi \in I$

(2) $A, B \in I$ imply $A \cup B \in I$

(3) $A \in I, B \subset A$ imply $B \in I$.

while an admissible ideal $I$ of $Y$ further satisfies $\{x\} \in I$ for each $x \in Y$. Given $I \subset 2^{\mathbb{N} \times \mathbb{N} \times \mathbb{N}}$ be a non trivial ideal in $\mathbb{N} \times \mathbb{N} \times \mathbb{N}$ and $(X, \mu, \eta, *, \delta)$ be an IFMS. A sequence $\left(x_{m n}\right)_{m, n, k \in \mathbb{N} \times \mathbb{N} \times \mathbb{N}}$ in $X$ is said to be $I$ - convergent to $0 \in X$ with respect to the intuitionistic fuzzy metric $(\mu, \eta)$ if for each $\epsilon>0$ and $t>0$ the set

$A(\epsilon)=\left\{m, n \in \mathbb{N} \times \mathbb{N} \times \mathbb{N}: \mu\left(x_{m n k}-0, t,\left\|\left(d_{1}\left(x_{1}\right), \ldots, d_{n}\left(x_{n}\right)\right)-0\right\|_{p}\right) \geq 1-\epsilon\right\}$ belongs to 
I. or

$A(\epsilon)=\left\{m, n \in \mathbb{N} \times \mathbb{N} \times \mathbb{N}: \eta\left(x_{m n k}-0, t,\left\|\left(d_{1}\left(x_{1}\right), \ldots, d_{n}\left(x_{n}\right)\right)-0\right\|_{p}\right) \leq \epsilon\right\}$ belongs to $I$.

2.6. Definition. A non-empty family of sets $F \subset 2^{X \times X \times X}$ is a filter on $X$ if and only if

(1) $\phi \in F$

(2) for each $A, B \in F$, we have imply $A \bigcap B \in F$

(3) each $A \in F$ and each $A \subset B$, we have $B \in F$.

2.7. Definition. An ideal $I$ is called non-trivial ideal if $I \neq \phi$ and $X \notin I$. Clearly $I \subset$ $2^{X \times X \times X}$ is a non-trivial ideal if and only if $F=F(I)=\{X-A: A \in I\}$ is a filter on $X$.

2.8. Definition. A non-trivial ideal $I \subset 2^{X \times X \times X}$ is called (i) admissible if and only if $\{\{x\}: x \in X\} \subset I$. (ii) maximal if there cannot exists any non-trivial ideal $J \neq I$ containing $I$ as a subset.

If we take $I=I_{f}=\{A \subseteq \mathbb{N} \times \mathbb{N} \times \mathbb{N}: A$ is a finite subset $\}$. Then $I_{f}$ is a non-trivial admissible ideal of $\mathbb{N}$ and the corresponding convergence coincides with the usual convergence. If we take $I=I_{\delta}=\{A \subseteq \mathbb{N} \times \mathbb{N} \times \mathbb{N}: \delta(A)=0\}$ where $\delta(A)$ denote the asyptotic density of the set $A$. Then $I_{\delta}$ is a non-trivial admissible ideal of $\mathbb{N} \times \mathbb{N} \times \mathbb{N}$ and the corresponding convergence coincides with the statistical convergence.

Let $D$ denote the set of all closed and bounded intervals $X=\left[x_{1}, x_{2}, x_{3}\right]$ on the real line $\mathbb{R} \times \mathbb{R} \times \mathbb{R}$. For $X, Y, Z \in D$, we define $X \leq Y \leq Z$ if and only if $x_{1} \leq y_{1} \leq z_{1}, x_{2} \leq y_{2} \leq z_{2}$ and $x_{3} \leq y_{3} \leq z_{3}, d(X, Y)=\max \left\{\left|x_{1}-y_{1}-z_{1}\right|,\left|x_{2}-y_{2}-z_{2}\right|\right\}$, where $X=\left[x_{1}, x_{2}, x_{3}\right]$ and $Y=\left[y_{1}, y_{2}, y_{3}\right]$.

Then it can be easily seen that $d$ defines a metric on $D$ and $(D, d)$ is a complete metric space. Also the relation $\leq$ is a partial order on $D$. A fuzzy number $X$ is a fuzzy subset of the real line $\mathbb{R} \times \mathbb{R} \times \mathbb{R}$ i.e. a mapping $X: R \rightarrow J(=[0,1])$ associating each real number $t$ with its grade of membership $X(t)$.

2.9. Definition. A fuzzy number $X$ is said to be (i) convex if $X(t) \geq X(s) \wedge X(r)=$ $\min \{X(s), X(r)\}$, where $s<t<r$. (ii) normal if there exists $t_{0} \in \mathbb{R} \times \mathbb{R} \times \mathbb{R}$ such that $X\left(t_{0}\right)=1$. (iii) upper semi-continuous if for each $\epsilon>0, X^{-1}([0, a+\epsilon])$ for all $a \in[0,1]$ is open in the usual topology of $\mathbb{R} \times \mathbb{R} \times \mathbb{R}$.

Let $R(J)$ denote the set of all fuzzy numbers which are upper semicontinuous and have compact support, i.e. if $X \in \mathbb{R}(J) \times \mathbb{R}(J) \times \mathbb{R}(J)$ the for any $\alpha \in[0,1],[X]^{\alpha}$ is compact, where $[X]^{\alpha}=\{t \in \mathbb{R} \times \mathbb{R} \times \mathbb{R}: X(t) \geq \alpha$, if $\alpha \in[0,1]\}$, $[X]^{0}=$ closure of $(\{t \in \mathbb{R} \times \mathbb{R} \times \mathbb{R}: X(t)>\alpha$, if $\alpha=0\})$.

The set $\mathbb{R}$ of real numbers can be embedded $\mathbb{R}(J)$ if we define $\bar{r} \in \mathbb{R}(J) \times \mathbb{R}(J) \times \mathbb{R}(J)$ by 


$$
\bar{r}(t)= \begin{cases}1, & \text { if } t=r: \\ 0, & \text { if } t \neq r\end{cases}
$$

The absolute value, $|X|$ of $X \in \mathbb{R}(J)$ is defined by

$$
|X|(t)= \begin{cases}\max \{X(t), X(-t)\}, & \text { if } t \geq 0 \\ 0, & \text { if } t<0\end{cases}
$$

Define a mapping $\bar{d}: \mathbb{R}(J) \times \mathbb{R}(J) \times \mathbb{R}(J) \rightarrow \mathbb{R}^{+} \cup\{0\}$ by

$$
\bar{d}(X, Y)=\sup _{0 \leq \alpha \leq 1} d\left([X]^{\alpha},[Y]^{\alpha},[Z]^{\alpha}\right) .
$$

It is known that $(\mathbb{R}(J), \bar{d})$ is a complete metric space.

2.10. Definition. A metric on $\mathbb{R}(J)$ is said to be translation invariant if $\bar{d}(X+Y, Y+Z)=$ $\bar{d}(X, Z)$, for $X, Y, Z \in \mathbb{R}(J)$.

2.11. Definition. Let $(X, \mu, \eta, *, \delta)$ be an IFMS and a sequence $X=\left(X_{m n k}\right)$ of fuzzy numbers is said to be convergent to a fuzzy number $X_{0}$ if for every $\epsilon>0$ and $t>0$, there exists a positive integer $n_{0}$ such that $\bar{d}\left(\mu\left(X_{m n k}, X_{0}, t\right)\right) \geq \epsilon$ or $\bar{d}\left(\eta\left(X_{m n k}, X_{0}, t\right)\right) \leq \epsilon$ for all $m, n, k \geq n_{0}$.

2.12. Definition. Let $(X, \mu, \eta, *, \delta)$ be an IFMS and a sequence $X=\left(X_{m n k}\right)$ of fuzzy numbers is said to be (i) $I$-convergent to a fuzzy number $X_{0}$ if for each $\epsilon>0$ and $t>0$ such that

$$
\begin{gathered}
A=\left\{m, n, k \in \mathbb{N}: \bar{d}\left(\mu\left(X_{m n k}, X_{0}, t\right)\right) \geq \epsilon\right\} \text { in } I \text { or } \\
A=\left\{m, n, k \in \mathbb{N}: \bar{d}\left(\eta\left(X_{m n k}, X_{0}, t\right)\right) \leq \epsilon\right\} \text { in } I
\end{gathered}
$$

The fuzzy number $X_{0}$ is called $I$-limit of the sequence $\left(X_{m n k}\right)$ of fuzzy numbers and we write $I-\lim X_{m n k}=X_{0}$. (ii) I-bounded if there exists $M>0$ such that

$$
\left\{m, n, k \in \mathbb{N}: d\left(X_{m n k}, \overline{0}\right)>M\right\} \in I .
$$

2.13. Definition. Let $(X, \mu, \eta, *, \delta)$ be an IFMS and a sequence space $E_{F}$ of fuzzy numbers is said to be (i) solid ( or normal) if $\left(Y_{m n k}\right) \in E_{F}$ whenever $\left(X_{m n k}\right) \in E_{F}$ and $\bar{d}\left(Y_{m n k}, \overline{0}\right) \leq$ $\bar{d}\left(X_{m n k}, \overline{0}\right)$ for all $m, n, k \in \mathbb{N}$. (ii) symmetric if $\left(X_{m n k}\right) \in E_{F}$ implies $\left(X_{\pi(m n k)}\right) \in E_{F}$ where $\pi$ is a permutation of $\mathbb{N} \times \mathbb{N} \times \mathbb{N}$.

Let $K=\left\{m_{1} n_{1} k_{1}<m_{2} n_{2} k_{2}<\ldots\right\} \subseteq \mathbb{N}$ and $E$ be a sequence space. A $K$-step space of $E$ is a sequence space

$$
\lambda_{m n k}^{E}=\left\{\left(X_{m_{p} n_{p} k_{p}}\right) \in w^{3}:\left(m_{p} n_{p} k_{p}\right) \in E\right\} .
$$

A canonical preimage of a sequence $\left\{\left(X_{m_{p} n_{p} k_{p}}\right)\right\} \in \lambda_{m n k}^{E}$ is a sequence $\left\{Y_{m n k}\right\} \in w^{3}$ defined as 


$$
Y_{m n k}= \begin{cases}X_{m n k}, & \text { if } m, n, k \in E \\ 0, & \text { otherwise }\end{cases}
$$

A canonical preimage of a step space $\lambda_{m n k}^{E}$ is a set of canonical preimages of all elements in $\lambda_{m n k}^{E}$, i.e. $y$ is in canonical preimage of $\lambda_{m n k}^{E}$ if and only if $Y$ is canonical preimage of some $x \in \lambda_{m n k}^{E}$.

2.14. Definition. Let $(X, \mu, \eta, *, \delta)$ be an IFMS and a sequence space $E_{F}$ is said to be monotone if $E_{F}$ contains the canonical pre-images of all its step spaces.

The following well-known inequality will be used throughout the article. Let $p=\left(p_{m n k}\right)$ be any sequence of positive real numbers with $0 \leq p_{m n k} \leq \sup _{m n k} p_{m n k}=G, D=\max \{1,2 G-1\}$ then

$\left|a_{m n k}+b_{m n k}\right|^{p_{m n k}} \leq D\left(\left|a_{m n k}\right|^{p_{m n k}}+\left|b_{m n k}\right|^{p_{m n k}}\right)$ for all $m, n, k \in \mathbb{N}$ and $a_{m n k}, b_{m n k} \in \mathbb{C}$.

Also $\left|a_{m n k}\right|^{p_{m n k}} \leq \max \left\{1,|a|^{G}\right\}$ for all $a \in \mathbb{C}$.

First we procure some known results; those will help in establishing the results of this article.

2.15. Lemma. Let $(X, \mu, \eta, *, \delta)$ be an IFMS and a sequence space $E_{F}$ is normal implies $E_{F}$ is monotone. (For the crisp set case, one may refer to Kamthan and Gupta [26, page 53).

2.16. Lemma. (Kostyrko et al., [30], Lemma 5.1). If $I \subset 2^{\mathbb{N} \times \mathbb{N} \times \mathbb{N}}$ is a maximal ideal, then for each $A \subset \mathbb{N}$ we have either $A \in I$ or $\mathbb{N}-A \in I$.

2.17. Definition. Let $d$ be a mapping from $R(I) \times R(I) \times R(I)$ into $R^{*}(I) \times R^{*}(I) \times R^{*}(I)$ and let the mappings $L, f:[0,1] \times[0,1] \times[0,1] \rightarrow[0,1] \times[0,1] \times[0,1]$ be symmetric, non-decreasing Musielak Orlicz in both arguments and satisfy $L \times L \times L(0,0,0)=0$ and $f \times f \times f(1,1,1)=1$. Denote $[d(X, Y, Z)]_{\alpha}=\left[\lambda_{\alpha}(X, Y, Z),(X, Y, Z)\right]$, for $X, Y \in R(I) \times$ $R(I) \times R(I)$ and $0<\alpha<1$.

The $(R(I) \times R(I) \times R(I), d, L \times L \times L, f \times f \times f)$ is called a fuzzy $p$ - metric space and $d$ a fuzzy translation metric, if

(1) $d(X, Y)=\overline{0}$ if and only if $X=Y=Z$,

(2) $d(X, Y)=d(Y, Z) d(Z, X)$ for all $X, Y, Z \in X$,

(3) for all $X, Y, Z \in R(I) \times R(I) \times R(I)$,

(i) $d(X, Y, Z)(s+t+u) \geq L \times L \times L(d(X, Y)(s), d(Y, Z)(t), d(Z, X)(u))$ whenever $s \leq$ $\lambda_{1}(X, Y), t \leq \lambda_{1}(Y, Z), u \leq \lambda_{1}(Z, X)$ and $(s+t+u) \leq \lambda_{1}(X, Y, Z)$,

(ii) $d(X, Y)(s+t+u) \leq f \times f \times f(d(X, Y)(s), d(Y, Z)(t), d(Z, X)(u))$ whenever $s \geq$ $\lambda_{1}(X, Y), t \geq \lambda_{1}(Y, Z), u \geq \lambda_{1}(Z, X)$ and $(s+t+u) \leq \lambda_{1}(X, Y, Z)$, 


\section{Some NeW Intuitionistic SEQUence SPACES of FuZZY numbers}

The main aim of this article to introduce the following sequence spaces and examine topological and algebraic properties of the resulting sequence spaces. Let $(X, \mu, \eta, *, \delta)$ be an IFMS and $p=\left(p_{m n k}\right)$ be a sequence of positive real numbers for all $m, n, k \in \mathbb{N} . f=\left(f_{m n k}\right)$ be a Musielak-Orlicz function, $\left(X,\left\|\left(d\left(x_{1}\right), d\left(x_{2}\right), \cdots, d\left(x_{n-1}\right)\right)\right\|_{p}\right)$ be a fuzzy $p$-metric space, and $\alpha_{m n k}(X)=\mu\left(\left((m+n+k) ! X_{m n k}\right)^{1 / m+n+k}, \overline{0}, t\right)=$

$\eta\left(\left((m+n+k) ! X_{m n k}\right)^{1 / m+n+k}, \overline{0}, t\right)$ be a sequence of fuzzy numbers. Using the concept of fuzzy metric, we introduce the following class of sequence:

$$
\begin{aligned}
& {\left[\chi_{f(\mu, \eta)}^{3 I(F)},\left\|\left(d\left(x_{1}\right), d\left(x_{2}\right), \cdots, d\left(x_{n-1}\right)\right)\right\|_{p}\right]=} \\
& \left\{(m, n, k) \in \mathbb{N} \times \mathbb{N} \times \mathbb{N}:\left[f_{m n k}\left(\left\|\alpha_{m n k}(x),\left(d\left(x_{1}\right), d\left(x_{2}\right), \cdots, d\left(x_{n-1}\right)\right)\right\|_{p}\right)\right] \leq 1-\epsilon\right\} \in I,
\end{aligned}
$$

3.1. Theorem. Let $f=\left(f_{m n k}\right)$ be a Musielak-Orlicz function, $(X, \mu, \eta, *, \delta)$ be an IFMS and the sequence space $\left[\chi_{f(\mu, \eta)}^{3 I(F)},\left\|\left(d\left(x_{1}\right), d\left(x_{2}\right), \cdots, d\left(x_{n-1}\right)\right)\right\|_{p}\right]$ is a linear space.

Proof: It is trivial. Therefore omit the proof.

3.2. Theorem. Let $(X, \mu, \eta, *, \delta)$ be an IFMS and the sequence spaces and the class of sequences $\left[\chi_{f(\mu, \eta)}^{3 I(F)},\left\|\left(d\left(x_{1}\right), d\left(x_{2}\right), \cdots, d\left(x_{n-1}\right)\right)\right\|_{p}\right]$ is solid and as such as monotone

Proof: Consider two sequences $\left(X_{m n k}\right)$ and $\left(Y_{m n k}\right)$ such that $\left|X_{m n k}\right| \leq\left|Y_{m n k}\right|$, for all $m, n, k \in \mathbb{N}$ and $Y_{m n k} \in\left[\chi_{f(\mu, \eta)}^{3 I(F)},\left\|\left(d\left(x_{1}\right), d\left(x_{2}\right), \cdots, d\left(x_{n-1}\right)\right)\right\|_{p}\right]$. We have

$\alpha_{m n k}(X)<\alpha_{m n k}(Y) \rightarrow \overline{0}$, as $m, n, k \rightarrow \infty$.

$\Rightarrow \alpha_{m n k}(X) \in\left[\chi_{f(\mu, \eta)}^{3 I(F)},\left\|\left(d\left(x_{1}\right), d\left(x_{2}\right), \cdots, d\left(x_{n-1}\right)\right)\right\|_{p}\right]$. Thus the class

$\left[\chi_{f(\mu, \eta)}^{3 I(F)},\left\|\left(d\left(x_{1}\right), d\left(x_{2}\right), \cdots, d\left(x_{n-1}\right)\right)\right\|_{p}\right]$ is solid. The class of sequences

$\left[\chi_{f(\mu, \eta)}^{3 I(F)},\left\|\left(d\left(x_{1}\right), d\left(x_{2}\right), \cdots, d\left(x_{n-1}\right)\right)\right\|_{p}\right]$ is monotone follows from the Lemma 2.15 .

3.3. Theorem. Let $(X, \mu, \eta, *, \delta)$ be an IFMS and the class of sequence $\left[\chi_{f(\mu, \eta)}^{3 I(F)},\left\|\left(d\left(x_{1}\right), d\left(x_{2}\right), \cdots, d\left(x_{n-1}\right)\right)\right\|_{p}\right]$ is not convergence free.

Proof: Consider a sequence $\left(X_{m n k}\right) \in\left[\chi_{f(\mu, \eta)}^{3 I(F)},\left\|\left(d\left(x_{1}\right), d\left(x_{2}\right), \cdots, d\left(x_{n-1}\right)\right)\right\|_{p}\right]$ defined as follows: For $m, n, k$ are even

$$
\alpha_{m n k}(X)=\left\{\begin{array}{l}
1+(m n k)^{3} t, \text { for }-(m n k)^{-3} \leq t \leq 0, \\
1-(m n k)^{3} t, \text { for } 0 \leq t \leq(m n k)^{-3}, \\
0, \text { otherwise }
\end{array}\right.
$$

and for $m, n, k$ are odd, $\alpha_{m n k}(X)=\overline{0}$.

Now for $\alpha \in(0,1]$, 


$$
\alpha_{m n k}(X)^{\gamma}=\left\{\begin{array}{l}
{\left[(\gamma-1)(m n k)^{-3},(1-\gamma)(m n k)^{-3}\right], \text { for } m, n, k \text { even }} \\
{[0,0], \text { for } m, n, k \text { odd } .}
\end{array}\right.
$$

Then $\alpha_{m n k}(X)=(\gamma-1)(m n k)^{-3} \rightarrow \overline{0}$, as $m, n, k \rightarrow \infty$, and

$\alpha_{m n k}(X)=(1-\gamma)(m n k)^{-3} \rightarrow \overline{0}$, as $m, n, k \rightarrow \infty$. Thus,

$\left(X_{m n k}\right) \in\left[\chi_{f(\mu, \eta)}^{3 I(F)},\left\|\left(d\left(x_{1}\right), d\left(x_{2}\right), \cdots, d\left(x_{n-1}\right)\right)\right\|_{p}\right]$. Let us define a sequence $\left(Y_{m n k}\right)$ as follows For $m, n, k$ are even

$$
\alpha_{m n k}(Y)=\left\{\begin{array}{l}
1+(m n k)^{1} t, \text { for }-(m n)^{-1} \leq t \leq 0 \\
1-(m n k)^{1} t, \text { for } 0 \leq t \leq(m n k)^{-1} \\
0, \text { otherwise }
\end{array}\right.
$$

and for $m, n, k$ are odd, $\alpha_{m n k}(Y)=\overline{0}$.

Now for $\alpha \in(0,1]$,

$$
\alpha_{m n k}(Y)^{\gamma}=\left\{\begin{array}{l}
{\left[(\gamma-1)(m n k)^{-1},(1-\gamma)(m n k)^{-1}\right], \text { for } m, n, \text { keven }} \\
{[0,0], \text { for } m, n, k \text { odd } .}
\end{array}\right.
$$

Then $\alpha_{m n k}(Y)=(\gamma-1)(m n k)^{-3} \not \supset \overline{0}$, as $m, n, k \rightarrow \infty$, and

$\alpha_{m n k}(Y)=(1-\gamma)(m n k)^{-3} \not \rightarrow \overline{0}$, as $m, n, k \rightarrow \infty$. Thus,

$\left(Y_{m n k}\right) \notin\left[\chi_{f(\mu, \eta)}^{3 I(F)},\left\|\left(d\left(x_{1}\right), d\left(x_{2}\right), \cdots, d\left(x_{n-1}\right)\right)\right\|_{p}\right]$. Hence

$\left[\chi_{f(\mu, \eta)}^{3 I(F)},\left\|\left(d\left(x_{1}\right), d\left(x_{2}\right), \cdots, d\left(x_{n-1}\right)\right)\right\|_{p}\right]$ is not convergence free.

3.4. Theorem. Let $(X, \mu, \eta, *, \delta)$ be an IFMS and the class of sequence $\left[\chi_{f(\mu, \eta)}^{3 I(F)},\left\|\left(d\left(x_{1}\right), d\left(x_{2}\right), \cdots, d\left(x_{n-1}\right)\right)\right\|_{p}\right]$ is symmetric

Proof: Let $\left(X_{m n k}\right) \in\left[\chi_{f(\mu, \eta)}^{3 I(F)},\left\|\left(d\left(x_{1}\right), d\left(x_{2}\right), \cdots, d\left(x_{n-1}\right)\right)\right\|_{p}\right]$. Let $\left(Y_{m n k}\right)$ be a arrangement of the sequence $\left(X_{m n k}\right)$ such that $X_{m n k}=Y_{p_{m} q_{n} r_{k}}$ for each $m, n, k \in \mathbb{N}$. Then $\alpha_{m n k}(X)=$ $\alpha_{m n k}(Y)$, as $m, n, k \rightarrow \infty$, and $\alpha_{m n k}(X)=\alpha_{m n k}(Y) \rightarrow \overline{0}$, as $m, n, k \rightarrow \infty$. Thus, $\left(Y_{m n k}\right) \in\left[\chi_{f(\mu, \eta)}^{2 I(F)},\left\|\left(d\left(x_{1}\right), d\left(x_{2}\right), \cdots, d\left(x_{n-1}\right)\right)\right\|_{p}\right]$.

Hence the class $\left[\chi_{f(\mu, \eta)}^{3 I(F)},\left\|\left(d\left(x_{1}\right), d\left(x_{2}\right), \cdots, d\left(x_{n-1}\right)\right)\right\|_{p}\right]$ is symmetric.

3.5. Theorem. Let $(X, \mu, \eta, *, \delta)$ be an IFMS and the class of sequence $\left[\chi_{f(\mu, \eta)}^{3 I(F)},\left\|\left(d\left(x_{1}\right), d\left(x_{2}\right), \cdots, d\left(x_{n-1}\right)\right)\right\|_{p}\right]$ is a sequence algebra

Proof: Let $\left(X_{m n k}\right),\left(Y_{m n k}\right) \in\left[\chi_{f(\mu, \eta)}^{3 I(F)},\left\|\left(d\left(x_{1}\right), d\left(x_{2}\right), \cdots, d\left(x_{n-1}\right)\right)\right\|_{p}\right]$,

then we have $\alpha_{m n k}(X) \rightarrow \overline{0}$, as $m, n, k \rightarrow \infty$ and $\alpha_{m n k}(Y) \rightarrow \overline{0}$, as $m, n, k \rightarrow \infty$, The result follows from the following inequalities $\alpha_{m n k}(X \otimes Y) \leq \alpha_{m n k}(X) \alpha_{m n k}(Y) \rightarrow \overline{0}$, as $m, n, k \rightarrow \infty$. Thus, $\alpha_{m n k}(X \otimes Y) \in\left[\chi_{f(\mu, \eta)}^{3 I(F)},\left\|\left(d\left(x_{1}\right), d\left(x_{2}\right), \cdots, d\left(x_{n-1}\right)\right)\right\|_{p}\right]$.

Hence the class $\left[\chi_{f(\mu, \eta)}^{3 I(F)},\left\|\left(d\left(x_{1}\right), d\left(x_{2}\right), \cdots, d\left(x_{n-1}\right)\right)\right\|_{p}\right]$ is sequence algebra. 
3.6. Theorem. (i)Let $(X, \mu, \eta, *, \delta)$ be an IFMS and if the sequence $\left(r_{m n k}\right)$ satisfies $\Delta_{2}-$ condition, then

$\left[\chi_{f(\mu, \eta, r)}^{3 I(F)},\left\|\left(d\left(x_{1}\right), d\left(x_{2}\right), \cdots, d\left(x_{n-1}\right)\right)\right\|_{p}\right]=\left[\chi_{f(\mu, \eta, s)}^{3 I(F)},\left\|\left(d\left(x_{1}\right), d\left(x_{2}\right), \cdots, d\left(x_{n-1}\right)\right)\right\|_{p}\right]$

(ii)Let $(X, \mu, \eta, *, \delta)$ be an IFMS and if the sequence $\left(s_{m n k}\right)$ satisfies $\Delta_{2}-$ condition, then $\left[\chi_{f(\mu, \eta, s)}^{3 I(F)},\left\|\left(d\left(x_{1}\right), d\left(x_{2}\right), \cdots, d\left(x_{n-1}\right)\right)\right\|_{p}\right]=\left[\chi_{f(\mu, \eta, r)}^{3 I(F)},\left\|\left(d\left(x_{1}\right), d\left(x_{2}\right), \cdots, d\left(x_{n-1}\right)\right)\right\|_{p}\right]$

Proof: Let $(X, \mu, \eta, *, \delta)$ be an IFMS and if the sequence $\left(r_{m n k}\right)$ satisfies $\Delta_{2}-$ condition, we get

$$
\left[\chi_{f(\mu, \eta, s)}^{3 I(F)},\left\|\left(d\left(x_{1}\right), d\left(x_{2}\right), \cdots, d\left(x_{n-1}\right)\right)\right\|_{p}\right] \subset\left[\chi_{f(\mu, \eta, r)}^{3 I(F)},\left\|\left(d\left(x_{1}\right), d\left(x_{2}\right), \cdots, d\left(x_{n-1}\right)\right)\right\|_{p}\right]
$$

To prove the inclusion

$\left[\chi_{f(\mu, \eta, r)}^{3 I(F)},\left\|\left(d\left(x_{1}\right), d\left(x_{2}\right), \cdots, d\left(x_{n-1}\right)\right)\right\|_{p}\right] \subset\left[\chi_{f(\mu, \eta, s)}^{3 I(F)},\left\|\left(d\left(x_{1}\right), d\left(x_{2}\right), \cdots, d\left(x_{n-1}\right)\right)\right\|_{p}\right]$

let $a \in\left[\chi_{f(\mu, \eta, r)}^{3 I(F)},\left\|\left(d\left(x_{1}\right), d\left(x_{2}\right), \cdots, d\left(x_{n-1}\right)\right)\right\|_{p}\right]$. Then for all $\alpha_{m n k}(X)$ with $\alpha_{m n k}(X) \in$ $\left[\chi_{f(\mu, \eta, r)}^{3 I(F)},\left\|\left(d\left(x_{1}\right), d\left(x_{2}\right), \cdots, d\left(x_{n-1}\right)\right)\right\|_{p}\right]$. We have

$$
\sum_{m=1}^{\infty} \sum_{n=1}^{\infty} \sum_{k=1}^{\infty}\left|x_{m n k} a_{m n k}\right|<\infty
$$

Since $(X, \mu, \eta, *, \delta)$ be an IFMS and the sequence $\left(r_{m n k}\right)$ satisfies $\Delta_{2}-$ condition, then $\alpha_{m n k}(Y) \in\left[\chi_{f(\mu, \eta, r)}^{3 I(F)},\left\|\left(d\left(x_{1}\right), d\left(x_{2}\right), \cdots, d\left(x_{n-1}\right)\right)\right\|_{p}\right]$, we get $\sum_{m=1}^{\infty} \sum_{n=1}^{\infty} \sum_{k=1}^{\infty}\left|\frac{y_{m n k} a_{m n k}}{(m+n+k) !}\right|<\infty$. by (3.2). Thus $\left(a_{m n k}\right) \in\left[\chi_{f(\mu, \eta, r)}^{3 I(F)},\left\|\left(d\left(x_{1}\right), d\left(x_{2}\right), \cdots, d\left(x_{n-1}\right)\right)\right\|_{p}\right]=\left[\chi_{f(\mu, \eta, s)}^{3 I(F)},\left\|\left(d\left(x_{1}\right), d\left(x_{2}\right), \cdots, d\left(x_{n-1}\right)\right)\right\|_{p}\right]$, and hence $\left(a_{m n k}\right) \in\left[\chi_{f(\mu, \eta, s)}^{3 I(F)},\left\|\left(d\left(x_{1}\right), d\left(x_{2}\right), \cdots, d\left(x_{n-1}\right)\right)\right\|_{p}\right]$. This gives that

$$
\left[\chi_{f(\mu, \eta, r)}^{3 I(F)},\left\|\left(d\left(x_{1}\right), d\left(x_{2}\right), \cdots, d\left(x_{n-1}\right)\right)\right\|_{p}\right] \subset\left[\chi_{f(\mu, \eta, s)}^{3 I(F)},\left\|\left(d\left(x_{1}\right), d\left(x_{2}\right), \cdots, d\left(x_{n-1}\right)\right)\right\|_{p}\right]
$$

we are granted with (3.1) and (3.3)

$\left[\chi_{f(\mu, \eta, r)}^{3 I(F)},\left\|\left(d\left(x_{1}\right), d\left(x_{2}\right), \cdots, d\left(x_{n-1}\right)\right)\right\|_{p}\right]=\left[\chi_{f(\mu, \eta, s)}^{3 I(F)},\left\|\left(d\left(x_{1}\right), d\left(x_{2}\right), \cdots, d\left(x_{n-1}\right)\right)\right\|_{p}\right]$

(ii) Similarly, one can prove that

$\left[\chi_{f(\mu, \eta, s)}^{3 I(F)},\left\|\left(d\left(x_{1}\right), d\left(x_{2}\right), \cdots, d\left(x_{n-1}\right)\right)\right\|_{p}\right]=\left[\chi_{f(\mu, \eta, r)}^{3 I(F)},\left\|\left(d\left(x_{1}\right), d\left(x_{2}\right), \cdots, d\left(x_{n-1}\right)\right)\right\|_{p}\right]$ if the sequence $\left(s_{m n k}\right)$ satisfies $\Delta_{2}-$ condition.

\section{Competing Interests:}

The authors declare that there is no conflict of interests regarding the publication of this research paper. 


\section{ACKNOWLEDGEMENT}

The authors are extremely grateful to the anonymous learned referee(s) for their keen reading, valuable suggestion and constructive comments for the improvement of the manuscript.

\section{REFERENCES}

[1] T. Apostol, Mathematical Analysis, Addison-Wesley, London, 1978.

[2] A. Esi, On some triple almost lacunary sequence spaces defined by Orlicz functions, Res. Rev., Discrete Math. Struct., 1(2) (2014), 16-25.

[3] A. Esi and M. Necdet Catalbas, Almost convergence of triple sequences, Glob. J. Math. Anal., 2(1) (2014), 6-10.

[4] A. Esi and E. Savas, On lacunary statistically convergent triple sequences in probabilistic normed space, Appl. Math. Inf. Sci., 9(5) (2015), 2529-2534.

[5] A. Esi, Statistical convergence of triple sequences in topological groups, Ann. Univ. Craiova, Math. Comput. Sci. Ser., 40(1) (2013), 29-33.

[6] E. Savas and A. Esi, Statistical convergence of triple sequences on probabilistic normed space, Ann. Univ. Craiova, Math. Comput. Sci. Ser., 39(2) (2012), 226-236.

[7] G.H. Hardy, On the convergence of certain multiple series, Proc. Camb. Phil. Soc., 19 (1917), 86-95.

[8] N. Subramanian and U.K. Misra, Characterization of gai sequences via double Orlicz space, Southeast Asian Bull. Math., 35 (2011), 687-697.

[9] N. Subramanian, C. Priya and N. Saivaraju, The $\int \chi^{2 I}$ of real numbers over Musielak metric space, Southeast Asian Bull. Math., 39 (1) (2015), 133-148.

[10] N. Subramanian, P. Anbalagan and P. Thirunavukkarasu, The Ideal Convergence of Strongly of $\Gamma^{2}$ in p- Metric Spaces Defined by Modulus, Southeast Asian Bull. Math., 37 (2013), 919-930.

[11] N. Subramanian, The Semi Normed space defined by Modulus function, Southeast Asian Bull. Math., 32 (2008), 1161-1166.

[12] Deepmala, N.Subramanian and Vishnu Narayan Misra, Double almost $\left(\lambda_{m} \mu_{n}\right)$ in $\chi^{2}-$ Riesz space, Southeast Asian Bull. Math., 35(2016), 1-11.

[13] N. Subramanian, B.C. Tripathy and C. Murugesan, The double sequence space of $\Gamma^{2}$, Fasciculi Math., 40 (2008), 91-103.

[14] N. Subramanian, B.C. Tripathy and C. Murugesan, The Cesáro of double entire sequences, Int. Math. Forum, 4(2)(2009), 49-59.

[15] A. Sahiner, M. Gurdal and F.K. Duden, Triple sequences and their statistical convergence, Selcuk J. Appl. Math., 8(2)(2007), 49-55.

[16] N. Subramanian and A. Esi, The generalized triple difference of $\chi^{3}$ sequence spaces, Glob. J. Math. Anal., 3(2) (2015), 54-60.

[17] N. Subramanian and A. Esi, The study on $\chi^{3}$ sequence spaces, Songklanakarin J. Sci. Technol., communicated.

[18] N. Subramanian and A. Esi, Characterization of Triple $\chi^{3}$ sequence spaces, Math. Moravica, in press.

[19] N. Subramanian and A. Esi, Some New Semi-Normed Triple Sequence Spaces Defined By A Sequence Of Moduli, J. Anal. Number Theory, 3(2) (2015), 79-88. 
[20] T.V.G. Shri Prakash, M. Chandramouleeswaran and N. Subramanian, The Triple Almost Lacunary $\Gamma^{3}$ sequence spaces defined by a modulus function, Int. J. Appl. Eng. Res., 10(80) (2015), 94-99.

[21] T.V.G. Shri Prakash, M. Chandramouleeswaran and N. Subramanian, The triple entire sequence defined by Musielak Orlicz functions over $p$ - metric space, Asian J. Math. Comput. Res., 5(4) (2015), 196-203.

[22] T.V.G. Shri Prakash, M. Chandramouleeswaran and N. Subramanian, The Random of Lacunary statistical on $\Gamma^{3}$ over metric spaces defined by Musielak Orlicz functions, Mod. Appl. Sci., 10(1) (2016), 171-183

[23] T.V.G. Shri Prakash, M. Chandramouleeswaran and N. Subramanian, The Triple $\Gamma^{3}$ of tensor products in Orlicz sequence spaces, Math. Sci. Int. Res. J., 4(2) (2015), 162-166.

[24] T.V.G. Shri Prakash, M. Chandramouleeswaran and N. Subramanian, The strongly generalized triple difference $\Gamma^{3}$ sequence spaces defined by a modulus, Math. Moravica, in press.

[25] T.V.G. Shri Prakash, M. Chandramouleeswaran and N. Subramanian, Lacunary Triple sequence $\Gamma^{3}$ of Fibonacci numbers over probabilistic $p-$ metric spaces, Int. Org. Sci. Res., in press.

[26] P.K. Kamthan and M. Gupta, Sequence spaces and series, Lecture notes, Pure and Applied Mathematics, 65 Marcel Dekker, Inc., New York, 1981.

[27] J. Lindenstrauss and L. Tzafriri, On Orlicz sequence spaces, Israel J. Math., 10 (1971), 379-390.

[28] H. Kizmaz, On certain sequence spaces, Canadian Math. Bull., 24(2)(1981), 169-176.

[29] H. Nakano Concave modulars, J. Math. Soc. Japan, 5, (1953), 29-49.

[30] P. Kostyrko, T. Salat and W.Wilczynski, I- convergence, Real Anal. Exchange, 26(2) (2000-2001), 669686.

[31] V.N. Mishra, Some Problems on Approximations of Functions in Banach Spaces, Ph.D. Thesis (2007), Indian Institute of Technology, Roorkee 247 667, Uttarakhand, India.

[32] V.N. Mishra, L.N. Mishra, Trigonometric Approximation of Signals (Functions) in $L_{p}(p \geq 1)$-norm, Int. J. Contemp. Math. Sci., 7 (19), 2012, 909-918.

[33] D. Rai, N. Subramanian, V.N. Mishra, The Generalized difference of $\int \chi^{2 I}$ of fuzzy real numbers over p- metric spaces defined by Musielak Orlicz function, New Trends Math. Sci., 4 (3) (2016), 296-306. 